\title{
Balkanologie
}

Balkanologie Revue d'études pluridisciplinaires

Vol. IX, n' $1-2 \mid 2005$

Volume IX Numéro 1-2

\section{Prise entre trois Romes. L'église de Grèce et les relations inter-ecclésiastiques dans la nouvelle Europe}

Vers un retour d'anciennes problématiques?

Entangled between 3 Romes. The orthodox Church of Greece and inter-

denominational Relations within a new European Framework: Towards a return of Old paradigms?

\section{Tassos Anastassiadis}

\section{CpenEdition}

\section{Journals}

Édition électronique

URL : http://journals.openedition.org/balkanologie/597

DOI : 10.4000/balkanologie. 597

ISSN : 1965-0582

Éditeur

Association française d'études sur les Balkans (Afebalk)

Édition imprimée

Date de publication : 1 décembre 2005

ISSN : 1279-7952

\section{Référence électronique}

Tassos Anastassiadis, « Prise entre trois Romes. L'église de Grèce et les relations inter-ecclésiastiques dans la nouvelle Europe », Balkanologie [En ligne], Vol. IX, n 1-2 | 2005, mis en ligne le 13 janvier 2010, consulté le 17 décembre 2020. URL : http://journals.openedition.org/balkanologie/597 ; DOI : https:// doi.org/10.4000/balkanologie.597 


\section{PRISE ENTRE TROIS ROMES : L'ÉGLISE DE GRĖCE ${ }^{1}$ ET LES RELATIONS INTER-ECCLÉSIASTIDUES DANS LA NOUVELLE EUROPE, VERS UN RETOUR D'ANCIENNES PROBLÉMATIQUES?}

Tassos Anastassiadis*

Il convient de commencer par poser une question, certes provocatrice, mais qui définit tout de même "l'horizon d'attente"2 des orthodoxes grecs ou, si on veut, des orthodoxies grecques : le schisme entre catholiques et orthodoxes se sera-t-il effacé à temps pour la commémoration de son millénaire en 2054 ?

Ce travail traite des récentes évolutions induites par le nouveau cadre européen dans le fonctionnement de l'Église de Grèce à la fois au niveau national et dans ses relations internationales. L'Église de Grèce est souvent présentée comme fondamentalement nationaliste et xénophobe, notamment depuis les années 1990 et en particulier l'accession au pouvoir de son primat actuel, l'archevêque Christodoulos, en 19983. Pour autant l'approche en termes d'une idéologie nationaliste conduit vite à une aporie. C'est aussi durant l'épiscopat de Monseigneur Christodoulos que l'Église a développé ses relations interna-

\footnotetext{
${ }^{1}$ Dans cet article, le terme "Église de Grèce" est utilisé à dessein et par commodité pour dèsigner l'Église orthodoxe autocéphale de Grèce.

• EHESS Paris - Universitè de Versailles Saint Quentin. Mail : TassosA@free.fr

${ }^{2}$ Nous utilisons cette expression dans le sens que lui donne Reinhard Koselleck dans son livre Koselleck (Reinhard), Le futur passé : contribution à la semantique des temps historiques, Paris : Éditions de l'EHESS, 1990 [1979], ch. 5, et en particulier p. 313 : " l'horizon, c'est cette ligne derrière laquelle va s'ouvrir un nouveau champ d'expérience dont on ne peut encore avoir connaissance".
}

3 Pollis (Adamantia), "Eastern Orthodoxy and Human Rights ", Human Rights Quarterly, 15 (2), mai 1993. Cf. le mème type d'affirmations dans lipowatz (Thanos), "Orthodoxos Christianismos kai Ethnikismos : dyo ptyhes tis syghronis Ellinikis politikis koultouras " (Christianisme orthodoxe et nationalisme : deux aspects de la culture politique grecque moderne), Elliniki Epitheorissi Politikis Epistimis, 2, 1993; Lipowatz est revenu sur la question dans un article intitulé Lpowatz (Thanos), "Logos kai thelisi ston Hristianismo " (Reason and Will in Christianism), Elliniki Epitheorissi Politikis Epistimis, 6, novembre 1995. Sur une appréciation de la periode après 1998 dans la longue durée, voir Diamandouros (Nikiforos), Politismikos dyismos kai politiki allagi stin Ellada tis metapolitefsis (Dualisme culturel et changement politique dans la Grèce post-autoritaire), Athènes : Alexandria, 2000. 
tionales et inter-ecclésiastiques de manière fulgurante 4 . Sommes nous en présence d'une schizophrénie dans l'action?

Nous espérons démontrer que la situation s'éclaircirait si nous quittions le domaine de l'explication idéologique pour se concentrer sur les aspects interactionnistes. Ici, les maitres mots, qui sont des classiques de l'histoire et de la sociologie des religions de Max Weber à Pierre Bourdieu ou encore Peter Brown seraient : activisme et militantisme dans un cadre de concurrence frénétique pour le monopole de la gestion des biens du salut 5 . Vus sous l'angle de l'activisme et du militantisme ecclésiastique, les deux niveaux (le niveau national et le niveau international) s'imbriquent et s'expliquent mutuellement. Ainsi pourra-t-on constater dans un premier temps, que l'activisme bien réel de l'Église grecque obéit à la même logique, aussi bien lorsqu'il se dirige contre les partisans de la sécularisation en Grèce que lorsqu'il accomplit des "miracles" en termes de rapprochement avec l'Église catholique. Ce constat mériterait ensuite d'être contextualisé. Cet activisme prend ses racines dans la prise en compte de l'évolution du contexte institutionnel : l'Église de Grèce, longuement seul représentant institutionnel orthodoxe au niveau européen, se trouve dans le cadre de la nouvelle Europe en situation à la fois de pluralisme institutionnel orthodoxe et de récepteur de sollicitations catholiques. Athènes doit désormais gérer des relations avec trois Romes ${ }^{6}$ et cela nécessite une certaine innovation stratégique. Toutefois, c'est aussi sur ce point qu'un rappel historique peut être utile afin de mettre en perspective cette innovation. La période de l'après 1917 nous offre à plusieurs titres une occasion saisissante de voir comment l'Église a déjà eu à faire face à ce type d'interrogations et comment elle a développé un répertoire d'actions, disponibles pour un réinvestissement permanent. Dressons d'abord le constat.

4 Pour un rappel de l'état des lieux de la recherche voir Anastassiadis (Tassos), " Religion and Politics in Greece : The Greek Church's "Conservative Modernization" in the 1990 ", Questions de Recherche du CERI, (11), janvier 2004.

5 Weber (Max), Économie et société, Paris : Agora, 1995 [1921],t. 2, chapitre V. Weber (Max), “ Les sectes protestantes et l'esprit du capitalisme ", in Weber (Max), L'éthique protestante et l'esprit du capitalisme, Paris : Agora, 1964 ; Bourdieu (Pierre), "Une interpretation de la théorie de la religion selon Max Weber ", Archives européennes de sociologie, 12 (1), 1971. Brown (Peter), Poverty and Leadership in the Late Roman Empire, Hanover : Brandeis University Press, 2002, en particulier, pp. 45-83.

${ }^{6}$ Par référence à Rome, Constantinople (la "Nouvelle Rome" depuis le transfert de la capitale de l'Empire romain en 330 ap. J.-C.) et Moscou (la "troisième Rome" depuis la chute de Constantinople en 1453). 


\section{LE CONSTAT : UN DOUBLE ACTIVISME EN APPARENCE CONTRADICTOIRE}

Il est important de souligner dans un premier temps que si nous suivions les commentateurs empêtrés dans le schéma dualiste simpliste Modernité/Tradition 7 nous nous trouverions face à un hiatus inexplicable entre le mode d'action (polémique) de l'Église par rapport à la société grecque et celui (plutôt coopératif) en relation avec les institutions supranationales/internationales ou même d'autres Églises. Prendre appui sur le refus par l'Église du principe de la crémation pour les orthodoxes ${ }^{8}$ ou encore de sa persistance à voir l'appartenance confessionnelle figurer sur les cartes d'identité grecques 9 pour démontrer son caractère "traditionnellement" conservateur et la nature nationaliste de son primat conduit vite à un paradoxe. Comment expliquer alors que c'est aussi sous Monseigneur Christodoulos qu'un évêque de Rome a pu visiter Athènes (en mai 2001) pour la première fois depuis le schisme entre christianisme oriental et occidental $^{10}$ ? En réalité ce paradoxe ne l'est qu'en apparence. Il a trait au double activisme de l'Église grecque (dirigé vers l'intérieur et l'extérieur).

Il est indiscutable que depuis l'accession au siège archiépiscopal de Monseigneur Christodoulos en 1998, l'Église de Grèce n'a cessé d'occuper une place centrale sur la scène médiatique et politique grecque ${ }^{11}$. Il ne se passe pas une semaine sans que ses déclarations ne fassent la une des journaux et deviennent un chiffon rouge pour divers éditorialistes, intellectuels et hommes politiques partisans d'une plus grande "sécularisation" en Grèce. Mais c'est surtout son activisme au niveau intérieur qui provoque l'ire des ses opposants. Cet activisme intérieur concerne, notamment, une plus grande implication au niveau de la bienfaisance et un meilleur quadrillage de la société par des insti-

7 La vision la plus sophistiquée de ce schéma en relation avec l'Église et la Grèce a été donnée par Diamandouros (Nikiforos), op. cit. Une première version en anglais de ce travail a été publiée sous le titre Cultural Dualism and Political Change in Postauthoritarian Greece, Madrid : Instituto Juan March, 1994.

${ }^{8}$ Voir sur ce sujet Dargentas (Magadalini), " Modern Greece ", in Davies (D.J.), éd., Encyclopedia of Cremation, Aldershot : Ashgate, 2005.

9 La littérature sur la question des cartes d'identité est très impressionnante. Pour un rapide tour d'horizon, voir le numéro spécial de la revue Social Compass édité par Vasilios Makrides et Lina MolokotosLiederman consacré à la Grèce : Social Compass, 51 (4), décembre 2004. Pour une approche essayant d'inscrire les controverses actuelles dans un cadre explicatif et non pas simplement descriptif et s'inscrivant dans la longue durée voir Anastassiadis (Tassos), art.cit.

${ }^{10}$ Sur les aspects évènementiels de la visite du Pape à Athènes voir de Montclos (Christine), "La visite du Pape à Athènes : une main tendue à l'Orthodoxie ", Cahiers d'Études sur la Méditerranée Orientale et le monde Turco-Iranien, (32), juillet-décembre 2001.

${ }^{11}$ Stavrakakis (Yannis), " Religious Populism and Political Culture : The Greek Case ", South European Society and Politics, 7 (3), hiver 2002. 
tutions de bienfaisance ecclésiastiques; la mesure qui servit de symbole et qui cristallisa d'ailleurs toutes les oppositions fut la décision de créer une allocation familiale destinée aux familles nombreuses orthodoxes des régions frontalières de la Grèce ${ }^{12}$. Afin de développer ce domaine, l'Église a voulu aussi valoriser la propriété ecclésiastique, ce qui a provoqué des conflits avec les collectivités locales qui auraient bien aimé voir le statut de cette propriété rester en suspens ${ }^{13}$. Souhaitant procéder à une simplification des structures ecclésiastiques orthodoxes au profit d'une structure pyramidale sous les ordres de l'archevēque de Grèce et du Synode, Monseigneur Christodoulos a également essayé d'affirmer son contrōle sur les diocèses du nord de la Grèce qui continuent à dépendre en partie du Patriarcat œcuménique provoquant ainsi l'ire de ce dernier ainsi que des élites laïques qui considèrent le patriarche œcuménique Bartholomé $\mathrm{I}^{\mathrm{er}}$ comme un représentant plus convenable de l'orthodoxie grecque ${ }^{14}$. Tout dernièrement, l'Église de Grèce semble avoir obtenu de la part du gouvernement la prise de contrôle de la formation des prêtres grâce à l'élévation des écoles ecclésiastiques en écoles de statut universitaire (alors que jusqu'à présent seules les facultés de théologie où on entrait par examen public assumaient ce rôle) ${ }^{15}$. Enfin, au niveau de la communication et de la visibilité, Monseigneur Christodoulos a mis en place la radio et la chaine de télévision ecclésiastiques tout en dynamisant la présence de l'Église sur la Toile ${ }^{16}$.

L'ensemble de ces axes d'action de l'Église témoigne bien de la volonté de la rendre plus impliquée dans la société grecque, pourvue d'un personnel plus motivé et plus fidèle à une hiérarchie centralisée. Ce qui apparaît en filigrane, c'est le désir de se renforcer en privilégiant la participation militante, plutôt que la pratique ou l'affiliation routinières, dans un environnement perçu comme hostile ou indifférent.

\footnotetext{
${ }^{12}$ Anastassiadis (Tassos), art.cit. Voir aussi le rapport d'étape d'un projet en cours sur l'effort de bienfaisance de l'Église de Grèce : Fokas (Effie), “ Orthodoxy, National Welfare and National Identity ", commu-

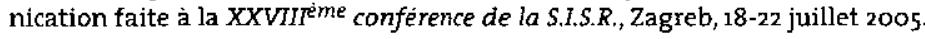

13 Voir les articles du quotidien Eleftherotypia du 6 février, du 22 et 24 avril et du 6 octobre 2004, concernant notamment une proprièté foncière à la région de Karea à Athènes.

14 Voir les articles publiés tous les jours dans le quotidien Eleftherotypia durant la période du 16 au 27 avril 2004 lorsque l'on était aux bords de la rupture et durant la période allant du 9 au 12 août 2004 lorsque l'on est parvenu à une "réconciliation" entre les deux protagonistes. Pour les élites laïques, le Patriarche Bartholomé représente une image de l'orthodoxie à la fois supranationale - et non pas étroitement nationale voire nationaliste - et modeme puisqu'il s'intéresse tout particulièrement aux questions de l'environnement - d'où son surnom de "Patriarche vert". Voir sur ce point le livre d'un ex-ministre du précédent gouvernement socialiste qui illustre parfaitement ce choix des milieux intellectuels de gauche : Siphounakis (Nikos), Politiki : shediasmos kai praxi (Politique : planification et action), Athènes : Kastaniotis, 2006.

15 Sur cette question voir notamment les interventions des universitaires Yorghos Sotirelis et Ioannis Tarnanidis dans le quotidien Eleftherotypia respectivement le 26 septembre et 10 octobre 2005.
}

${ }^{16}$ Voir le très performant site de l'Église grecque : 〈www.ecclesia.gr〉 qui inclut une bibliothèque, des archives synodales et une librairie en ligne. 
La même logique conduit à un activisme tout différent au niveau extérieur. À ce niveau, on privilégie la recherche d'alliances et de partenaires, alors que l'inertie avait dominé depuis bien longtemps. Le rapprochement avec l'Église catholique (visite du Pape en 2001, multiplication des partenariats avec différentes institutions romaines ${ }^{17}$ ) décidée elle aussi à se battre contre les partisans de la "laïcisation" à l'extérieur et de l'accommodation à l'intérieur n'est pas innocent. De même, c'est depuis Monseigneur Christodoulos que l'Église de Grèce participe de manière institutionnelle à un dialogue intense avec le groupe de la démocratie chrétienne européenne (le PPE). Il y a déjà eu huit dialogues depuis 1996 entre le PPE et les institutions orthodoxes. Le neuvième dialogue a eu lieu à Istanbul en octobre 2005 ; or, il convient de rappeler que Monseigneur Christodoulos avait été le représentant de l'Église de Grèce lors du premier dialogue alors qu'il n'était qu'une étoile montante du champ ecclésiastique grec ${ }^{18}$. Enfin c'est aussi Monseigneur Christodoulos qui a finalement ouvert un bureau de représentation à Bruxelles et ce, dès son accession au siège athénien en $1998^{19}$. L'Église de Grèce n'en possédait pas, alors que la Grèce fait partie de la CEE depuis 1981.

Ce sont justement les évolutions au niveau du cadre européen qui nous fournissent les premiers indices quant à la compréhension des dynamiques en cours.

\section{AMORCE D'UNE EXPLICATION : LE CONTEXTE EUROPÉEN}

Pendant longtemps la Grèce a été le seul pays de la CEE avec une forte Église orthodoxe, qui plus est établie. De même, l'affaiblissement du Patriarcat œcuménique en Turquie et la situation complexe du Patriarcat moscovite sous le régime soviétique aurait dû faire d'Athènes le seul véritable interlocuteur. Mais ce ne fut pas le cas. L'Église de Grèce s'était montrée indifférente et la représentation était assumée par le Patriarcat œcuménique. Or, depuis 1989 et la chute du Mur de Berlin, la deuxième Rome (Istanbul/Constantinople) et la troisième Rome (Moscou) furent de nouveau propulsées au devant de la scène, ce qui suscita des inquiétudes du côté athénien.

La fin de l'URSS donna au Patriarcat moscovite une plus grande marge d'autonomie mais surtout stimula les mêmes phénomènes centrifuges qu'on

${ }^{17}$ Ainsi, et ce à titre d'exemple, la Bibliothèque apostolique vaticane et le Service de Mission intérieure de l'Église de Grèce ont lancé un ambitieux projet conjoint d'édition de manuscrits byzantins et/ou orthodoxes se trouvant dans la Bibliothèque vaticane.

18 Sur l'historique de ces dialogues et leurs comptes-rendus, voir le site du PPE :<http://www.epped.org/Activities/pcurrentissues/politics-religion/orthodox-churches_en.asp>.

19 Voir le site de la représentation : 〈http://www.regue.org〉. 
constata au niveau politique. Souhaitant s'émanciper de la tutelle russe, toute une série d'Églises orthodoxes de l'ex-URSS (Géorgie, Ukraine) s'est rapprochée de la seule source de légitimité ecclésiastique orthodoxe "supérieure", le Patriarcat œcuménique. La réinstauration en 1996 de l'Église d'Estonie par le Patriarcat œcuménique provoqua même une passagère rupture des relations entre Constantinople et Moscou $^{20}$.

Le renouveau du Patriarcat constantinopolitain fut accentué par l'évolution des frontières de l'UE. En 1995, la Finlande rejoignit l'UE, ajoutant une deuxième Église orthodoxe établie. Bien que rassemblant peu de fidèles, cette dernière avait une valeur symbolique considérable. Elle permettait au Patriarcat constantinopolitain dont dépendent à la fois l'Église de Finlande, mais aussi l'Église sémi-autonome de Crète, certains diocèses de Grèce et les exarchats des communautés orthodoxes d'Europe occidentale de revendiquer avec force le statut de pôle orthodoxe et de veritable représentant des orthodoxes au sein de l'Union ${ }^{21}$.

L'accession à l'Union de Chypre et de l'Estonie a renforcé ce projet. Les candidatures de la Bulgarie et de la Roumanie ne pourront aller que dans ce sens également. Ce n'est pas non plus un hasard si le Patriarcat soutient ardemment l'entrée de la Turquie. Ses partisans ne manqueront pas de faire remarquer que face à l'Église catholique qui avance unie, seul le Patriarcat constantinopolitain dispose d'une légitimité suffisante pour être l'unique et donc efficace représentant des Églises orthodoxes, dont la fragmentation constitue aussi un handicap dans le cadre de l'ingénierie institutionnelle européenne. Pour paraphraser la célèbre phrase de Kissinger, on pourrait dire "l'orthodoxie, quel numéro de téléphone ?"

Le Patriarcat se verrait bien donc dans le rôle d'un représentant et superviseur d'une confédération des Églises orthodoxes qui pourrait mieux aborder les discussions avec l'Église catholique au sein de l'UE. Mais cette compétition pour la représentation a bien évidemment suscité l'inquiétude de l'Église de Grèce. D'unique représentant des orthodoxes, elle risque fort de se retrouver complètement marginalisée. Cette crainte la motive à la fois pour se renforcer en interne, réduire le poids patriarcal en son sein, et construire des nouvelles alliances inter-ecclésiastiques.

C'est tout naturellement qu'elle s'est tournée vers ceux qui risquaient d'être les autres grands perdants d'un renforcement de la deuxième Rome, voir d'un rapprochement intense entre les deux premières Romes : Moscou. Les relations

\footnotetext{
20 Voir une présentation faite de cette crise mais aussi de la volonté des Églises orthodoxes non-russes de l'Est de s'affranchir de Moscou dans le site officiel de l'Église orthodoxe d'Estonie : <http://www.orthodoxa.org/FR/estonie/HistoireEglise/sommhistoirEglise.htm>.
}

${ }^{21}$ Sur les territoires sous la juridiction du Patriarcat voir son site : 〈http://www.ec-patr.gr/patrdisplay php?lang=en\&id=5>. 
entre Athènes et Moscou sont au beau fixe depuis quelques années, ce qui constitue une nouveauté depuis le XIX ${ }^{\text {ème }}$ siècle.

Pour cette même raison on se montre attentif aux signaux de l'Église de Rome en direction des orthodoxes. Il faut sur ce point dire, que celle-ci gère pour l'instant la situation avec beaucoup de diplomatie. Certes, le Pape JeanPaul II a visité Athènes (et a souhaité visiter Moscou) mais il a aussi procédé à un acte symbolique fort en rendant au Patriarche Bartholomé Ier lors d'une cérémonie commune au Vatican les reliques de deux de ses anciens et plus illustres prédécesseurs : Grégoire de Nazianze et Jean Chrysostome. Et ce, alors qu'on commémorait justement le $800^{\text {ème }}$ anniversaire du sac de Constantinople par la quatrième croisade ${ }^{22}$.

Aussi nouvelle que puisse être la conjoncture, il ne faut pas pour autant croire que l'Église grecque innove complètement. Les Églises sont des institutions attachées à leur généalogie et abhorrant l'idée d'innovation. Tout changement, car le changement est inévitable, doit donc être justifié au nom d'un retour à la "Tradition". Il y avait déjà eu par le passé un moment où sur fond de profondes mutations sociales et géopolitiques en Europe, d'affaiblissement de l'Empire et de l'Église russes, d'efforts unionistes de l'Église catholique, Athènes et la Nouvelle Rome s'étaient affrontées sur la question de la représentation des orthodoxes. Les répertoires d'action s'étaient déjà mis en place à cette époque et ils pouvaient de nouveau être mobilisés et valorisés ${ }^{23}$.

\section{UN RETOUR PAR LE PASSÉ : LA PROBLÉMATIQUE DE L'UNION}

Lors de l'accession au siège pontifical de Benoît XVI au printemps 2005, les commentateurs furent nombreux à spéculer sur le choix de son nom. On s'attacha surtout à évoquer le rôle de Benoît XV (1914-1922) durant la Grande Guerre et aussi le rôle organisateur du fondateur de l'ordre des bénédictins Saint Benoît de Nursie ${ }^{24}$. Pour autant, nous pensons qu'il faut également, sinon davantage, regarder du côté du rôle de Benoît XV dans la définition de la politique du St. Siège en direction des orthodoxes, dans le cadre d'une vision d'union entre chrétientés catholique et orthodoxe en Europe.

${ }^{22}$ Voir le site de l'Église catholique à ce sujet : 〈http://www.vatican.va/news_services/liturgy/2004/documents/ns_lit_doc_20041127_index_fr.html>.

${ }^{23}$ Sur cette mobilisation des différentes facettes de "la Tradition" d'une Église voir l'analyse très classique de Halbwachs (Maurice), Les cadres sociaux de la mémoire, Paris : Albin Michel, 1994 [1925], en particulier pp. 190-204.

${ }^{24}$ L'allocution du 27 avril du nouveau Pape est allée dans ce sens. Voir : <http://www.generationjpii .org/article666.html>. 
Rappelons d'abord que la première sortie dominicale de Benoît XVI pour saluer la foule réunie dans la place St. Pierre fut effectuée le dimanche qui correspondait à la Pâques orthodoxe (1er mai 2005). Il consacra une substantielle partie de son message à l'attention des Orientaux qu'il salua " avec une affection particulière ${ }^{25}$. De même, son premier voyage pastoral en dehors de Rome l'amena à Bari pour l'Assemblée générale de la Conférence épiscopale italienne ${ }^{26}$. Le saint patron de cette ville n'est autre que Saint Nicolas, l'évêque grec de Myra, dont les reliques y furent transportées d'Orient au XI ${ }^{\text {ème }}$ siècle et qui est aussi un grand saint de l'Église orthodoxe. Se saisissant de cette symbolique, Benoît XVI martela sa volonté d'œuvrer en " faveur de l'unité des chrétiens " au sein d'une Église militante face à l'indifférence et la froideur du monde extérieur ${ }^{27}$. Il s'agit d'un discours qui cible la laïcisation comme le véritable adversaire de tous les chrétiens et qui ne peut déplaire aux prélats grecs.

Or, le fait que Benoit XVI se considère investi de la mission d'œuvrer en faveur de l'unité des chrétiens dans le cadre d'une Europe en pleine recomposition suite à la chute de l'Empire soviétique nous semble un indice sérieux quant au choix du nom. En effet, ce fut justement Benoît XV qui se trouva chargé de définir l'action catholique en direction des orthodoxes au lendemain de la Révolution d'octobre et de la chute de l'Empire russe en 1917. C'est en 1917 que furent créés le PIO (Pontificio Instituto Orientale), c'est-à-dire l'université romaine chargée des questions du christianisme oriental, et la Congrégation Pro Ecclesia Orientali (qui fut séparée de la célèbre Congrégation De Propaganda Fide). C'est à ce moment que la question des catholiques de rite byzantin (ou uniates) fut de nouveau remise à l'ordre du jour afin de réussir cette unité. Le sujet est vaste et mériterait un traitement à part ${ }^{28}$. Nous le mentionnons ici parce que c'est précisément dans la suite de ces événements et durant les années 1920 que l'Église grecque fut amenée à réfléchir sur la manière de jouer un nouveau rôle sur l'échiquier des Églises chrétiennes. La révolution bolchevique ayant paralysé l'Église russe, la défaite grecque à la guerre grécoturque de 1922 ayant affaibli le Patriarcat constantinopolitain, elle se voyait bien assumer le rôle de coordinatrice des Églises orthodoxes face aux tentati-

\footnotetext{
${ }^{25}$ htttp://www.vatican.va/holy_father/benedict_xvi/angelus/2005/documents/hf_ben-xvi_reg_ 20050501_workers_fr.html>.

26 <http://www.vatican.va/holy_father/benedict_xvi/angelus/2005/documents/hf_ben-xvi_ang_ 20050529_bari_fr.htmls.

27 <http://www.vatican.va/holy_father/benedict_xvi/homilies/2005/documents/hf_ben-xvi_hom_ 20050529_bari_fr.htmls.

${ }^{28}$ Sur ces questions voir deux travaux fondateurs de Croce (Mgr Giuseppe), " Alle origini della Congregazione orientali e del PIO : Il contributo de Mons. Louis Petit ", Orientalia Christiana Periodica, 53 . 1987 ; Ibid., "Monde hellène et chrétienté romaine : L'Union introuvable ", Ricerche di storia sociale e religiosa, 33 (66), juillet-décembre 2004 .
} 
ves d'union catholique. C'est avec vigueur qu'elle refusa à plusieurs reprises à l'époque les propositions de faire du Patriarcat un pôle d'allégeance supranational pour tous les orthodoxes ${ }^{29}$.

Elle se rendit compte que se maintenir impliquait deux voies d'action : à l'échelle européenne il fallait nouer des alliances inter-ecclésiastiques. À l'époque elle favorisa les projets œcuméniques avec les mainstream protestants ${ }^{3 \circ}$. Quant à l'échelle nationale, il fallait organiser un quadrillage efficace de "sa" société nationale grâce à une meilleure organisation bureaucratique et davantage hiérarchisée. Cette organisation se devait d'investir le domaine de l'action de bienfaisance face à la crise sociale de l'entre-deux-guerres. Elle devait également être plus militante aussi, s'attaquant à ce qui était perçu comme l'indifférence croissante des masses suite à la guerre. L'activisme de l'Église se devait être un Ianus à deux visages : militant agressif en interne; militant irénique en externe.

Comme dans les années 1920, l'Église de Grèce est aujourd'hui confrontée à un contexte géopolitique fluide; elle fait l'objet d'une sollicitation de la part de la première Rome; elle se trouve en situation d'antagonisme avec la deuxième ; elle constate les bouleversements qui secouent la troisième ; elle doit faire face à une nouvelle poussée à la fois du pluralisme religieux et de l'indifférence. Face à ces bouleversements et à la perspective de nouveaux efforts de réunification "chrétienne" en Europe, elle peut se retourner vers un répertoire d'action déjà rodé et réinvestissable. Cette démarche comporte l'avantage d'autoriser l'innovation tout en se montrant attaché à la tradition. Décidément, les voies des serviteurs du Seigneur sont bien impénétrables, ou presque...

\footnotetext{
${ }^{29}$ Sur ces sujets voir Anastassiadis (Tassos), " Religious Minorities Ecclesiastical Antagonism and Church Modernization: The Greek Orthodox Church, Past and Present ", communication faite à la XXVIIle conférence de la S.I.S.R., Zagreb, 18-22 juillet 2005 .

$3^{\circ}$ Voir Anastassiadis (Tassos), "Controverses politiques et tolérance canonique : la relecture au sein de l'Église orthodoxe grecque du $\mathrm{XX}^{\text {ème }}$ siècle de la notion patristique d'oikonomia et les relations avec les Anglicans n, Études Balkaniques, 10, 2003.
} 